\title{
Metabolomic analysis of the effects of edible dry beans (Phaseolus vulgaris L.) on tissue lipid metabolism and carcinogenesis in rats
}

\author{
Meghan M. Mensack ${ }^{1,2}$, John N. McGinley ${ }^{1}$ and Henry J. Thompson ${ }^{1 *}$ \\ ${ }^{1}$ Cancer Prevention Laboratory, Colorado State University, Fort Collins, CO, 80523, USA \\ ${ }^{2}$ Department of Chemistry, Colorado State University, Fort Collins, CO, 80523, USA \\ (Submitted 27 July 2011 - Accepted 16 September 2011)
}

\section{Abstract}

Metabolite profiling using liquid chromatography-time-of-flight MS was undertaken to identify candidate metabolic processes that account for dry bean effects on disease risk with a specific focus on the development of breast cancer. Normal mammary gland and mammary carcinomas from previously reported experiments were evaluated. Principal component analysis (PCA) of mass spectral data revealed that tissue of both types from control-fed $v$. bean-fed rats could be distinguished by their metabolomic profiles. Candidate ion identification using MassTRIX analysis software revealed that alterations in eicosanoid, fatty acid, TAG and steroid metabolism partially accounted for the differences observed in both PCA. In addition, evidence was obtained consistent with the hypothesis that the varying inhibitory effects on mammary carcinogenesis of genetically distinct dry bean types were mirrored by differential patterns of lipid metabolites in mammary carcinoma. The use of MassTRIX provided links for metabolite profile enrichment with metabolic pathways in the Kyoto Encyclopedia of Genes and Genomes. Implicated pathways included a linkage between diacylglycerol and protein kinase C and eicosanoid metabolites and inducible cyclo-oxygenase-2 and/or eicosanoid degradation mediated via 15-PG dehydrogenase. These pathways have been reported to be misregulated during the development of cancer. The differences observed between control-fed and bean-fed rats in lipid metabolism require validation using targeted analytical methods and detailed analyses of how bean bioactive food components regulate genes that control lipid biosynthesis, interconversion and catabolism.

\section{Key words: Dry beans (Phaseolus vulgaris L.): Metabolomics: Lipid metabolism: Breast cancer}

The edible dry bean, Phaseolus vulgaris L., is a pulse crop, i.e. a grain legume that serves as a staple food crop for populations around the world ${ }^{(1)}$. Since ancient times, pulses have been combined with cereal crops, primarily wheat, rice and corn, or tubers such as potato or cassava to provide a balanced source of protein and carbohydrate and a nutritionally adequate diet when combined with seed oils, fruit and vegetables ${ }^{(2,3)}$. In this setting, the established ratio of cereal grains to pulses to meet nutritional requirements is $2: 1$; however, over the last 50 years both production and consumption patterns, globally, have changed to $8: 1^{(1,4)}$. In developing countries, the consumption of pulses declines with a rise in socio-economic status since pulses crops, particularly dry beans, are considered 'poor man's

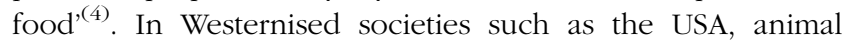
sources of protein have largely displaced pulse crops from the diet and even when individuals choose not to eat animal products, they frequently use soya-derived protein as the legume source ${ }^{(5)}$. While soya has many desirable properties, it is an oil-seed legume with markedly different profiles of macro- and micronutrients and bioactive food components compared with dry beans. While the pandemic of obesity that has swept the globe over the last 50 years is now receiving considerable attention ${ }^{(6,7)}$, as are the associated increases in the incidence of and mortality due to type 2 diabetes, heart disease and cancer, there has been virtually no scrutiny of the potential association between the decline in edible dry bean consumption and the global rise in obesity and its associated metabolic disorders $^{(8)}$. The work reported in the present paper represents a first step in addressing this issue at the molecular level.

A small but growing body of literature that was recently reviewed $^{(2,9)}$ indicates that increased consumption of dry beans is protective against the occurrence of type 2 diabetes, CVD and certain types of cancer. In addition, our laboratory and others have reviewed evidence that outlines the metabolic effects that are common among these diseases ${ }^{(8,10)}$. All three diseases are associated with alterations in glucose homeostasis,

Abbreviations: COD, centre of domestication; KEGG, Kyoto Encyclopedia of Genes and Genomes; LC, liquid chromatography; MPP, Mass Profiler Professional; NV, navy; PC, principal component; PCA, principal component analysis; SR, small red; WK, white kidney.

*Corresponding author: Dr Henry J. Thompson, fax +1970 491 3542, email henry.thompson@colostate.edu 
chronic inflammation and cellular oxidation ${ }^{(11,12)}$. What have been less emphasised are the alterations in lipid metabolism that also accompany these chronic diseases ${ }^{(13)}$. Relative to mechanisms of effect, there are a paucity of specific hypotheses about how dry beans exert protective effects. Recently, our laboratory reported that dry bean consumption inhibited the occurrence of experimentally induced breast cancer and provided evidence that dry bean intake affected both host systemic and cell autonomous mechanisms ${ }^{(14,15)}$. Evidence was also reported that showed dry bean varieties differed in anticancer activity in a manner that could be predicted by their genetic heritage $^{(14)}$. Moreover, several lines of evidence point to potential effects of dry beans on lipid metabolism in accounting for health benefits ${ }^{(9,16-18)}$. Consequently, the objective of the experiments reported in the present paper was to determine if the pattern of cellular metabolites detected in breast tissue harvested from tumour-free animals in our previously reported carcinogenesis experiments or in the mammary carcinomas that were induced in those experiments was consistent with the hypothesis that dry bean consumption alters lipid metabolism. Three questions were addressed: (1) how does dry bean consumption affect metabolite patterns in mammary gland from cancer-free animals and in the mammary carcinomas that occurred in tumour-bearing animals; (2) what pathways of cell regulation are inferred by the metabolite patterns; and (3) do the patterns of metabolites differ by dry bean genotype?

\section{Methods}

\section{Materials and chemicals}

The following chemicals and materials were used as received from Thermo Fisher: chloroform, methanol, acetonitrile and HPLC-grade water.

\section{Source of tissue for analysis}

The mammary gland and mammary carcinomas evaluated in the present study were obtained from a previously reported experiment $^{(14)}$. Briefly, female Sprague-Dawley rats were obtained from Taconic Farms at age $20 \mathrm{~d}$. Animal rooms were maintained at $22 \pm 2{ }^{\circ} \mathrm{C}$ with $50 \%$ relative humidity and a $12 \mathrm{~h}$ light-12 h dark cycle. At age $21 \mathrm{~d}$, rats were injected with 1-methyl-1-nitrosourea $(50 \mathrm{mg} / \mathrm{kg}$ body weight, intraperitoneally), as previously described. For week 1 of the study, rats were housed three per cage in solid-bottomed polycarbonate cages equipped with a food cup; they were given free access to American Institute of Nutrition (AIN)-93G control diet. At 7 $\mathrm{d}$ following carcinogen injection, all rats were randomised to diet groups based on body weight: control diet, small red (SR) bean, navy (NV) bean, or white kidney (WK) bean diet. Beans were incorporated at $60 \%(\mathrm{w} / \mathrm{w})$. Rats were fed their assigned diets ad libitum until the end of the study at $46 \mathrm{~d}$ post-carcinogen injection. The post-initiation design of this experiment simulates the promotion and progression events of the disease process, which is highly relevant to women at increased risk for breast cancer and to breast cancer survivors. The tissue was harvested from animals following an overnight fast of $12-14 \mathrm{~h}$. The work followed guidelines approved by the Colorado State University Animal Care and Use Committee.

Because of inter-individual variability in the metabolite profiles among animals, ten mammary glands from ten different rats per treatment group (WK, NV, SR, control) were used for analysis (200 mg tissue per sample). The mammary glands were taken from animals that had no palpable tumours. Similarly, ten carcinomas per treatment condition (200 mg tissue per sample) were also evaluated. Cancer incidence and multiplicity per treatment group were 95, 71, 66 and $63 \%$ and $3 \cdot 23$, $1.55,1.67$ and 1.14 carcinomas per rat for control, SR, NV and WK bean diet groups, respectively ${ }^{(14)}$. The 1-methyl-1-nitrosourea-induced model for breast cancer is one of the most widely used laboratory systems for studying this disease process in a pre-clinical setting. Histologically, the carcinomas induced and their pre-malignant precursors have many similarities to the human disease. A histological comparison of the lesions induced in this model relative to those observed in the human disease has been published by our laboratory ${ }^{(19)}$.

\section{Sample preparation}

Before extraction, the mammary gland and tumour tissue samples were ground under liquid $\mathrm{N}_{2}$ and $100 \mathrm{mg}$ of each sample placed in separate $1.5 \mathrm{ml}$ microcentrifuge tubes. Metabolite extraction was carried out using a modified Bligh and Dyer method published by Sana et al. ${ }^{(20)}$. To account for differential solubility of various compounds and to extract the largest number of compounds possible from the mammary and tumour tissue samples, extractions were carried out at $\mathrm{pH}$ 2 and $9^{(21)}$. Following extraction, samples were dried completely using a Speedvac under ambient temperature and stored at $-80^{\circ} \mathrm{C}$ until analysis. Before liquid chromatography (LC)MS analysis, samples were redissolved in $100 \mu \mathrm{l}$ 50:50 methanol-water ( $\mathrm{pH} 7$, milli-Q) by adding $50 \mu \mathrm{l}$ methanol followed by $50 \mu \mathrm{l}$ water with a brief vortexing step after each addition. A quantity of $900 \mu \mathrm{l}$ of a 75:25 mixture of acetonitrile-water was added at room temperature followed again by a brief vortexing step. Samples were placed in a $-20^{\circ} \mathrm{C}$ freezer for $1 \mathrm{~h}$, centrifuged and the supernatant fraction carefully removed from any pelleted material that was present, transferred to new $1.8 \mathrm{ml}$ tubes, and dried using a Speedvac. The samples were again redissolved in 50:50 methanol-water by adding $50 \mu \mathrm{l}$ methanol followed by $50 \mu \mathrm{l}$ Milli-Q water (containing $0 \cdot 4 \%$ $(\mathrm{v} / \mathrm{v})$ acetic acid) and vortexed after each solvent was added. The $100 \mu$ l was transferred to a fresh $1.8 \mathrm{ml}$ centrifuge tube containing $0 \cdot 2 \mu \mathrm{m}$ ultrafiltration membrane. Extracts were centrifuged at $4000 \mathrm{rpm}, 4^{\circ} \mathrm{C}$ for $20 \mathrm{~min}$. The liquid below the membrane was transferred to a tube containing a $10 \mathrm{kDa}$ filter, centrifuged at $4000 \mathrm{rpm}, 4^{\circ} \mathrm{C}$ for $20 \mathrm{~min}$ or until the majority of liquid had passed through the membrane. The acidic $(\mathrm{pH} 2)$ and basic $(\mathrm{pH}$ 9) samples were combined in equal volumes in LC vials for analysis.

\section{Sample analysis using liquid chromatography-MS}

Extracts were separated with a Zorbax SB-Aq column $2 \cdot 1 \times 150 \mathrm{~mm}$ on an Agilent $1200 \mathrm{LC}$. Injections $(5 \mu \mathrm{l})$ were 
made from $100 \mu \mathrm{l}$ sample volumes. A flow rate of $0.6 \mathrm{ml} / \mathrm{min}$ was used with a 2 to $98 \%$ linear gradient of water-methanol over 13 min followed by a 7 min solvent hold. A $0 \cdot 2 \%$ solution of acetic acid was used as a mobile phase modifier. An Agilent 6510 Quadrupole Time-of-Flight LC-MS with an electrospray ion source was used to acquire accurate-mass profiling MS data for tissue samples. Data were collected in both positive and negative ionisation modes over the range of 50 to $1500 \mathrm{~m} / z$.

\section{Multivariate analysis}

Initial data processing was carried out using the Molecular Feature Extraction algorithm in MassHunter Workstation software (Agilent Technologies). The retention time/mass pairs generated by MassHunter Workstation were exported as CEF files for subsequent analysis in Agilent Mass Profiler Professional (MPP) software. MPP was used for retention time and mass abundance adjustments. The data files were aligned to correct for retention time drift and raw intensities were used in statistical analysis. Any intensity less than 1 was set to 1 before analysis. Samples were imported into MPP using the guided workflow option. The following filter parameters were used for importing sample data: minimum absolute abundance was set at 1000 , minimum number of ions was set to 2 , retention time greater than $0.3 \mathrm{~min}$, and a mass between 50 and $1500 \mathrm{~m} / z$. Furthermore, only those features present in eight out of ten replicates, in one or more groups were analysed. The features were then analysed to determine differential features across groups using one-way ANOVA $(P<0 \cdot 05$, Benjamini-Hochberg false discovery rate (FDR) multiple testing correction) and KruskalWallis one-way $\mathrm{ANOVA}^{(22,23)}$. The resulting mass list was exported as a CEF file for recursive analysis. Datasets were re-mined using the Find by Formula algorithm in MassHunter with a mass window of 10 parts per million (ppm) and a retention time window of $0.2 \mathrm{~min}$. The general integrator was applied for recursive analysis. The resulting data files were re-imported into MPP for analysis using parameters identical to the original import described above. Unsupervised principal component (PC) analysis (PCA) with mean centring and scaling was carried out to visualise differences in metabolite profiles of the four treatment groups. Differential abundances of metabolites between WK bean-fed, NV bean-fed or SR bean-fed $v$. controlfed animals were determined using an unpaired $t$ test with Benjamini Hochberg FDR multiple test correction $(P<0 \cdot 05)^{(24)}$. Inclusion lists were generated for those features found to be at least 2-fold higher in all three bean-fed groups or those features found to be at least 2-fold higher in the control compared with all three bean-fed groups. Feature lists were queried against the Kyoto Encyclopedia of Genes and Genomes (KEGG; http:// www.genome.jp/kegg) using MassTRIX (http://masstrix.org) with a mass error of $7 \mathrm{ppm}$ and Rattus norvegicus as the model organism in order to assign tentative compound identities and active pathways.

\section{Results}

The effect of bean diets on the mammary gland metabolite profile

Using an unbiased analytical approach, i.e. not targeted to a specific class of chemicals, tissue samples were analysed in both positive and negative ionisation mode over the $\mathrm{m} / \mathrm{z}$ range of 50-1500. Within this $m / z$ range, 495 and 1775 features were detected in positive and negative ionisation modes, respectively. Applying a one-way ANOVA before PCA reduced these numbers to 201 and 253 features $(P<0 \cdot 05)$. As shown in Fig. 1(A), the control group is clearly separated from the bean diet groups in ESI-positive mode, with $95.42 \%$ of the total variance being explained by the first three components (PC1, $77 \cdot 11 \%$; PC2, 12.28\%; PC3, 6.03\%). Despite the large amount
(A)

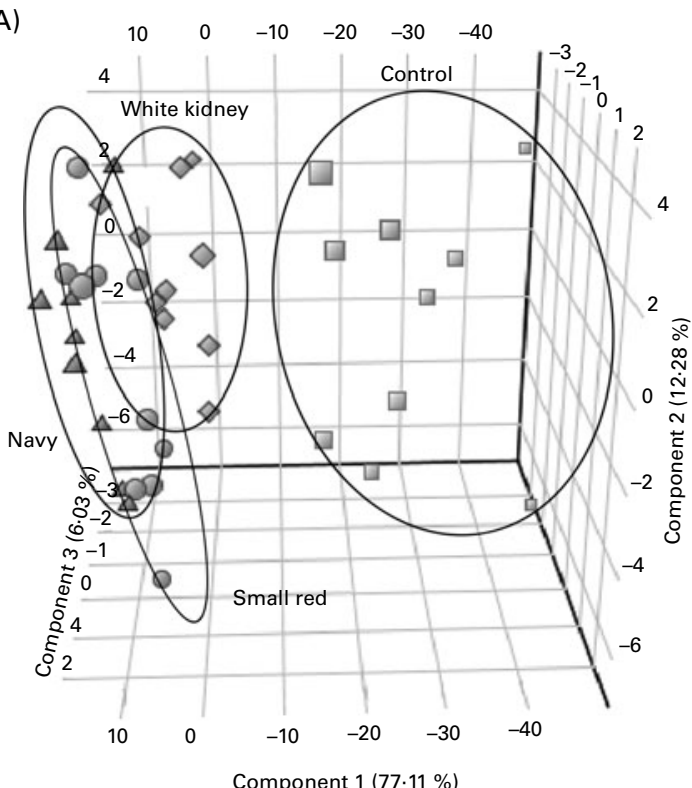

(B)

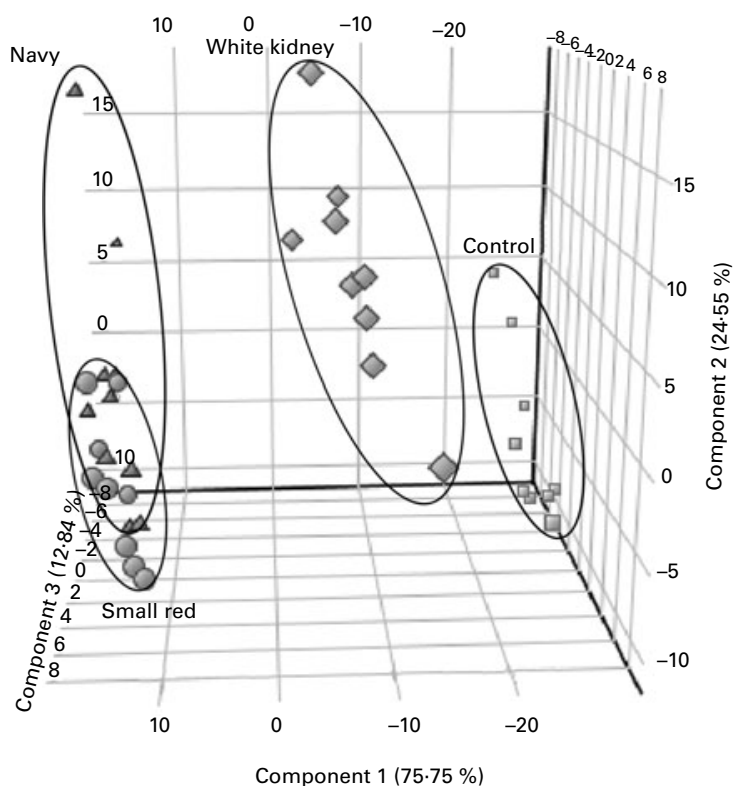

Fig. 1. Principal component analysis (PCA) scores plot for the comparison of mammary gland tissue from control and bean-fed rats based on (A) positive ionisation mode and $(B)$ negative ionisation mode. PCA shows a separate clustering based on control $(\square)$, white kidney $(\diamond)$, small red $(O)$ and navy $(\triangle)$ bean diets. 
of variation explained by the first three components, the three bean-fed groups are not clearly distinguishable based on the features detected in ESI-positive mode. It is also the case that the metabolites in ESI-negative mode clearly separate the control-fed animals from the bean-fed groups (Fig. 1(B)), where $55.73 \%$ of the total variance is explained by the first component, with PC2 and PC3 explaining an additional 24.55 and $12.84 \%$ of the total variance, respectively. In contrast to the metabolite profiles generated in ESI-positive mode, the WK bean-fed group is clearly distinguishable from both the control and the other two bean-fed groups. Of the 201 features detected in positive ionisation mode, 186 were overlapping in all three bean groups using control as a baseline, with only twenty-two being tentatively identified after submission to MassTRIX using $R$. norvegicus (brown rat) as the model organism (Table 1). In negative ionisation mode, 192 features were found at consistently higher levels in NV, SR and WK bean-fed animals when compared with the control group and nineteen ions were identified by mapping the metabolites to pathways in KEGG using MassTRIX (Table 1). Small molecules elevated in the control group were also explored. In this case, a total of thirty-nine molecules were elevated in the control-fed group relative to all three bean-fed groups (twelve and twenty-seven for positive and negative mode, respectively). Of these molecules, nine were tentatively identified using MassTRIX (Table 1).

\section{Bean diets down-regulate TAG synthesis in the mammary gland}

A total of five TAG precursors were tentatively identified using the KEGG database in all diet groups (Fig. 2). Of the five TAG precursors, three were significantly reduced in the mammary gland tissue of bean-fed animals when compared with the control group (Kruskal-Wallis; $P<0 \cdot 05$ ). The molecule putatively identified as palmitic acid was the most

Table 1. List of identified metabolites in mammary gland*

\begin{tabular}{|c|c|c|c|c|}
\hline $\mathrm{RT}(\min )$ & Mass (Da) & Mode & Trend† & Metabolite type $\ddagger$ \\
\hline 0.38 & $125 \cdot 0150$ & $\mathrm{ESI}-$ & $\uparrow$ & Amino acid \\
\hline 0.40 & 136.0372 & $\mathrm{ESI}-$ & $\downarrow$ & Amino acid \\
\hline $5 \cdot 13$ & $284 \cdot 1864$ & $\mathrm{ESI}+$ & $\downarrow$ & Secondary amine \\
\hline 7.04 & $255 \cdot 2562$ & $\mathrm{ESI}+$ & $\downarrow$ & Primary carboxylic acid amide \\
\hline $7 \cdot 66$ & 244.0697 & $\mathrm{ESI}+$ & $\downarrow$ & Primary alcohol \\
\hline $8 \cdot 20$ & $283 \cdot 2877$ & $\mathrm{ESI}+$ & $\downarrow$ & Primary amide \\
\hline $8 \cdot 80$ & $290 \cdot 1502$ & $\mathrm{ESI}+$ & $\uparrow$ & Eicosanoid \\
\hline $8 \cdot 87$ & $454 \cdot 3270$ & $\mathrm{ESI}+$ & $\downarrow$ & Primary alcohol \\
\hline $9 \cdot 20$ & $198 \cdot 1606$ & $\mathrm{ESI}+$ & $\uparrow$ & Unsaturated fatty acid \\
\hline $10 \cdot 47$ & $292 \cdot 2041$ & $\mathrm{ESI}-$ & $\uparrow$ & Hydroxy fatty acid \\
\hline $10 \cdot 62$ & $272 \cdot 2352$ & $\mathrm{ESI}-$ & $\downarrow$ & Hydroxy fatty acid \\
\hline $10 \cdot 95$ & $226 \cdot 1934$ & ESI - & $\downarrow$ & Unsaturated fatty acid \\
\hline $11 \cdot 15$ & $418 \cdot 2691$ & $\mathrm{ESI}+$ & $\downarrow$ & Secondary alcohol \\
\hline $11 \cdot 25$ & $276 \cdot 2088$ & ESI - & $\uparrow$ & Unsaturated fatty acid \\
\hline 11.58 & 654.4169 & ESI - & $\uparrow$ & Glycophosphocholine \\
\hline 11.58 & $440 \cdot 2767$ & ESI - & $\uparrow$ & Glycophosphocholine \\
\hline 11.59 & $360 \cdot 2290$ & ESI - & $\uparrow$ & Steroid lipid \\
\hline 11.59 & $278 \cdot 2259$ & $\mathrm{ESI}-$ & $\uparrow$ & Unsaturated fatty acid \\
\hline 11.59 & $346 \cdot 2124$ & $\mathrm{ESI}-$ & $\uparrow$ & Steroid lipid \\
\hline 11.59 & $862 \cdot 1912$ & $\mathrm{ESI}+$ & $\uparrow$ & Ketone \\
\hline 11.65 & $254 \cdot 2258$ & ESI - & $\downarrow$ & Unsaturated fatty acid \\
\hline 11.65 & $336 \cdot 2287$ & ESI - & $\downarrow$ & Prostaglandin F series \\
\hline 11.78 & $352 \cdot 2589$ & $\mathrm{ESI}+$ & $\downarrow$ & Primary alcohol \\
\hline $11 \cdot 88$ & $348 \cdot 2300$ & $\mathrm{ESI}+$ & $\downarrow$ & Ketone \\
\hline $12 \cdot 10$ & $270 \cdot 2561$ & $\mathrm{ESI}+$ & $\downarrow$ & Methyl branched fatty acid \\
\hline $12 \cdot 22$ & $890 \cdot 7303$ & $\mathrm{ESI}-$ & $\downarrow$ & TAG \\
\hline $12 \cdot 22$ & 662.4788 & $\mathrm{ESI}-$ & $\downarrow$ & Glycophosphocholine \\
\hline $12 \cdot 32$ & $332 \cdot 2719$ & $\mathrm{ESI}-$ & $\downarrow$ & Unsaturated fatty acid \\
\hline $12 \cdot 61$ & $298 \cdot 2877$ & $\mathrm{ESI}+$ & $\downarrow$ & Methyl branched fatty acid \\
\hline $12 \cdot 61$ & $284 \cdot 2714$ & $\mathrm{ESI}+$ & $\downarrow$ & Methyl branched fatty acid \\
\hline 13.05 & $646 \cdot 1599$ & $\mathrm{ESI}+$ & $\downarrow$ & Bile acids, alcohols and derivatives \\
\hline 13.08 & $598 \cdot 3104$ & $\mathrm{ESI}-$ & $\downarrow$ & Phosphoinositol \\
\hline $13 \cdot 16$ & $468 \cdot 3224$ & $\mathrm{ESI}+$ & $\downarrow$ & Vitamin $D_{2}$ and derivatives \\
\hline $13 \cdot 71$ & 586.4595 & $\mathrm{ESI}+$ & $\downarrow$ & Diacylglycerol \\
\hline $13 \cdot 79$ & $596 \cdot 5482$ & $\mathrm{ESI}-$ & $\uparrow$ & Steroid lipid \\
\hline $13 \cdot 80$ & 562.4623 & $\mathrm{ESI}+$ & $\downarrow$ & Diacylglycerol \\
\hline $13 \cdot 87$ & 608.4465 & $\mathrm{ESI}+$ & $\downarrow$ & Primary alcohol \\
\hline $14 \cdot 18$ & 652.6109 & ESI - & $\uparrow$ & Steroid lipid \\
\hline $14 \cdot 21$ & $618 \cdot 5193$ & $\mathrm{ESI}+$ & $\downarrow$ & Diacylglycerol \\
\hline 14.44 & 646.5509 & $\mathrm{ESI}+$ & $\downarrow$ & Diacylglycerol \\
\hline $14 \cdot 78$ & $720 \cdot 5706$ & $\mathrm{ESI}+$ & $\downarrow$ & Diacylglycerol \\
\hline
\end{tabular}

$\mathrm{RT}$, retention time; $\mathrm{ESI}$ - , electrospray ionisation negative mode; $\mathrm{ESI}+$, electrospray ionisation positive mode.

* List based on MassTRIX query of Kyoto Encyclopedia of Genes and Genomes (KEGG).

$\dagger$ Indicates feature is increased in bean-fed animals $(\uparrow)$ or decreased in bean-fed animals $(\downarrow)$ relative to the control group.

$\ddagger$ Metabolite type assigned based on KEGG search using MassTRIX. Total number of masses $=454$, metabolite types identified $=41 / 454$ or $9.0 \%$, non-identified metabolite types $=413 / 454$ or $91.0 \%$ (not shown). 


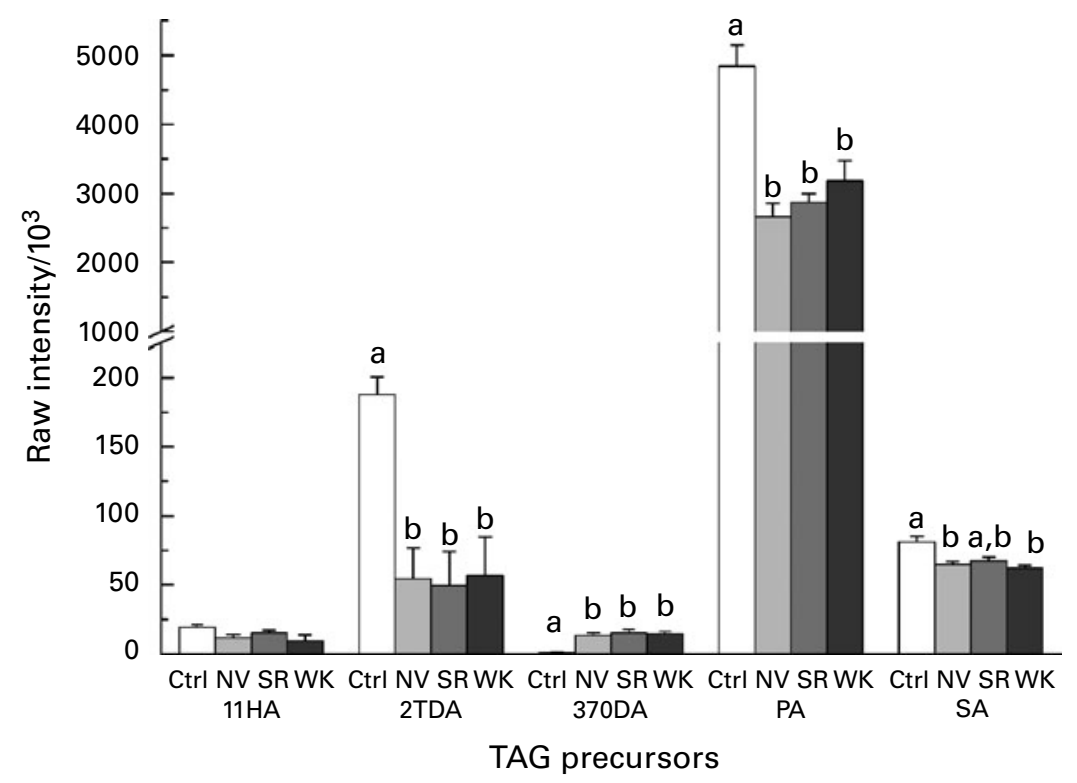

Fig. 2. Lipid metabolism in mammary gland tissue. Comparison of TAG precursors tentatively identified in mammary gland tissue. Values are means of at least nine animals per group ( $n 9-10)$, with standard errors represented by vertical bars. Metabolite intensity (relative concentration) was measured using liquid chromatography-time-of-flight MS and anlysed using Kruskal-Wallis one-way ANOVA. ${ }^{\text {a,b }}$ Mean values with unlike letters were significantly different $(P<0.05)$. Ctrl, control; NV, navy bean-fed; SR, small red bean-fed; WK, white kidney bean-fed; 11HA, 11-hydroxy palmitic acid; 2TDA, 2-tetradecenoic acid; 37ODA, 3,7-octadecadiynoic acid; PA, palmitic acid; SA, stearic acid.

abundant TAG precursor in all of the diet groups evaluated. 2-Tetradecenoic acid was also significantly reduced in all bean-fed animals while 3,7-octadecadiynoic acid was the only elevated TAG putatively identified in the three bean-fed groups. Stearic acid was found to be significantly reduced in only the NV bean-fed and WK bean-fed animals. The decrease in 11-hydroxypalmitic acid was not statistically significant in any of the three bean-fed groups.

The effect of bean diet on n- 6 fatty acids in the mammary gland

n-6 Fatty acids were compared between bean-fed and control animals. The compound tentatively identified as 18 : 3n-6 ( $\gamma$-linolenic acid) was elevated in the three bean-fed groups (Kruskal-Wallis; $P<0 \cdot 05$ ) while adrenic acid (all-cis-7,10,13, 16-docosatetraenoic acid) was reduced by more than 2 -fold in all three bean-fed groups (Fig. 3). The reduction of adrenic acid was significantly different between control and NV beanfed, SR bean-fed and WK bean-fed animals (Kruskal-Wallis; $P<0.05)$.

\section{The effect of bean diet on diacylglycerol levels in the mammary gland}

A total of five ions tentatively identified as diacylglycerols were reduced by the inclusion of dry edible beans in the diet (Fig. 4). Relative concentration of all five diacylglycerols was statistically different between each of the bean-fed groups and the controlfed animals (Kruskal-Wallis; $P<0 \cdot 05$ ). None of these molecules was statistically different between SR and NV bean-fed groups and only the diacylglycerols with masses of 586.4595 and
720.5706 Da were statistically different between WK and SR bean-fed $(P<0 \cdot 05)$ and WK and NV bean-fed $(P<0 \cdot 05)$ groups.

\section{The effect of bean diets on the mammary carcinoma metabolite profile}

As was the case in the mammary gland tissue, distinct clustering based on bean type is evident in the scores plot generated following unsupervised PCA in both positive (Fig. 5(A)) and negative (Fig. 5(B)) ionisation modes with the first component explaining the difference between the bean-fed groups and the control-fed group in both cases. The first three components in positive mode explain $95.18,2.09$ and $1.63 \%$ of the total variance in the dataset whereas the first three components in negative mode explain $61.02,23.1$ and $8.97 \%$ of the total variance. Distinct clustering of the samples based on bean type is shown by the PCA scores plots of both positive and negative ionisation modes. Of the 2119 features detected in positive ionisation mode, 379 were statistically different between the bean groups and control group (one-way ANOVA; $P<0.05$ ), with 327 higher in the bean-fed animals and just fifty-two higher in the control group. In contrast, of the 838 features detected in negative ionisation mode, all fifty-nine found to be statistically different between the bean-fed and control animals were higher in the control group. A total of twenty-eight features were tentatively identified after submission to MassTRIX using R. norvegicus (brown rat) as the model organism (Table 2).

\section{The effect of bean diet on fatty acids and fatty aldehyde compounds in mammary carcinomas}

Fatty acyl compounds were tentatively identified as features differentially regulated with the inclusion of beans in the diet 


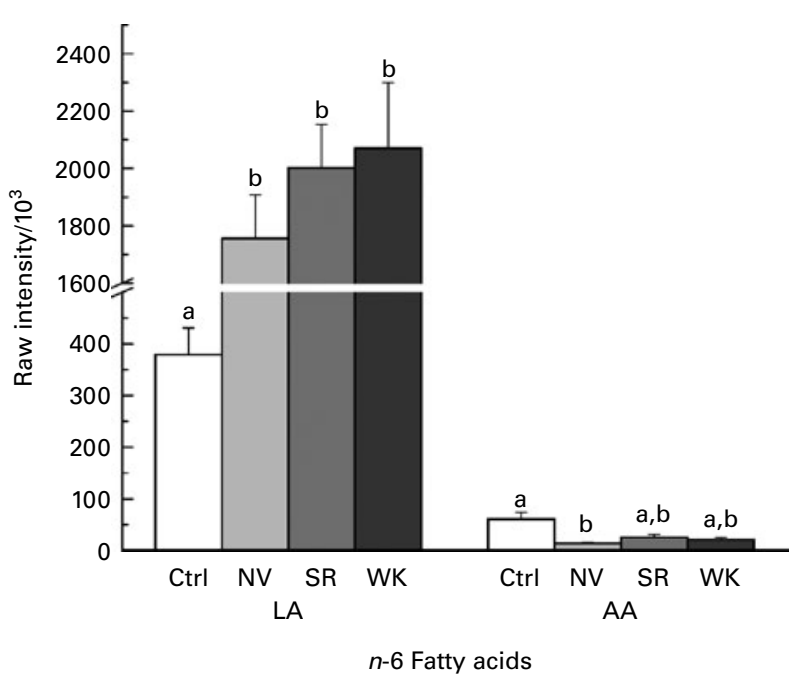

Fig. 3. $n-6$ Fatty acids tentatively identified in mammary gland tissue. Values are means of at least nine animals per group $(n 9-10)$, with standard errors represented by vertical bars. Metabolite intensity (relative concentration) was measured using liquid chromatography-time-of-flight MS and analysed using Kruskal-Wallis one-way ANOVA. ${ }^{a, b}$ Mean values with unlike letters were significantly different $(P<0.05)$. Ctrl, control; NV, navy bean-fed; SR, small red bean-fed; WK, white kidney bean-fed; LA, $18: 3 n-6$ ( $\gamma$-linolenic acid); $A A$, adrenic acid.

depending upon the subclass of molecule. Figure 6 illustrates this for (A) methyl branched fatty acids, (B) straight-chain fatty acids, (C) oxo fatty acids and (D) fatty aldehydes. Compounds with masses corresponding to methyl branched fatty acids (2,14-dimethyl hexanoic acid and isopalmitic acid) and the fatty aldehyde (9-octadecenal) were reduced in the bean-fed groups $(P<0 \cdot 05)$. In addition, 3,7,11-trimethyldocecanoic acid (methyl branched fatty acid) and the fatty aldehyde 2-tetradecenal were not detected in the bean-fed groups. In contrast, molecules putatively identified as straightchain fatty acids (3-capryl propionic acid, 8-oxo-nonanoic acid) and the oxo fatty acid caproic acid were not detected in the control group. The molecule tentatively identified as octanoic acid was significantly increased in all three bean groups (Kruskal-Wallis; $P<0.05$ ). Additionally, isopalmitic acid, 2,14-dimethyl hexanoic acid, caproic acid, octanoic acid, 3-capryl propionic acid, 8-oxo-nonanoic acid and 9-octadecenal were statistically different between WK and NV bean-fed groups as well as between WK and SR bean-fed animals (Kruskal-Wallis; $P<0 \cdot 05$ ). Interestingly, only isopalmitic acid was statistically different between the $\mathrm{NV}$ and SR bean-fed groups (Kruskal-Wallis; $P<0 \cdot 05$ ).

\section{Bean diet-induced changes in tumour prostaglandins}

Ions putatively identified as prostaglandins, in particular the $\mathrm{E}$ series, were up-regulated by bean diets. The increase in PGE3 was statistically significant (Kruskal-Wallis; $P<0.05$ ) when comparing the three bean-fed groups individually with the control. This particular prostaglandin mimics the cancer-prevention activity of the bean races, high cancer-prevention activity correlates with the larger increase in PGE3 (WK, 88521.667 (SEM 7680.379)), intermediate cancer-prevention activity correlates with an intermediate level of PGE3 (SR, 91011.714 (SEM 5906.439)) and the lowest cancer-prevention activity correlates with the lowest PGE3 level (NV, 101044.769 (sEm 6969.341)). Interestingly, PGE3 was significantly different between the WK and SR (Kruskal-Wallis, $P<0.05$ ) as well as between the WK and NV genotypes (Kruskal-Wallis; $P<0.05$ ) but not between the NV and SR genotypes (Kruskal-Wallis; $P=0.995$ ) (data not shown).

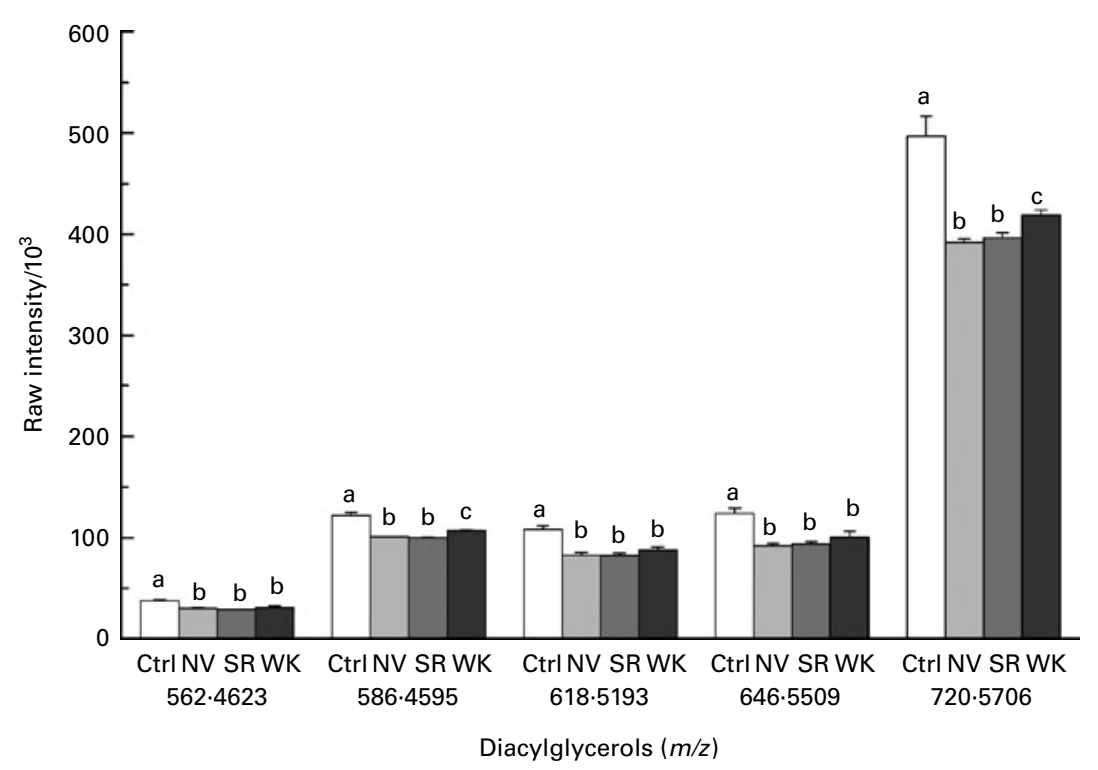

Fig. 4. Reduction of diacylglycerols in mammary gland as a result of bean feeding. The mass of each tentatively identified diacylglycerol is given below each set of four treatment groups. Values are means of at least nine animals per group $(n 9-10)$, with standard errors represented by vertical bars. Metabolite intensity (relative concentration) was measured using liquid chromatography-time-of-flight MS and analysed using Kruskal-Wallis one-way ANOVA. ${ }^{\text {a,b,c }}$ Mean values with unlike letters were significantly different $(P<0.05)$. Ctrl, control; NV, navy bean-fed; SR, small red bean-fed; WK, white kidney bean-fed. 
(A)

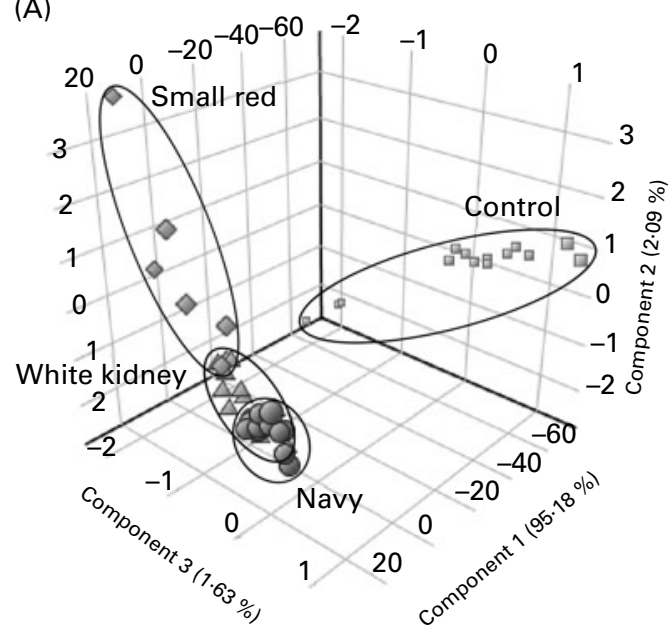

(B)

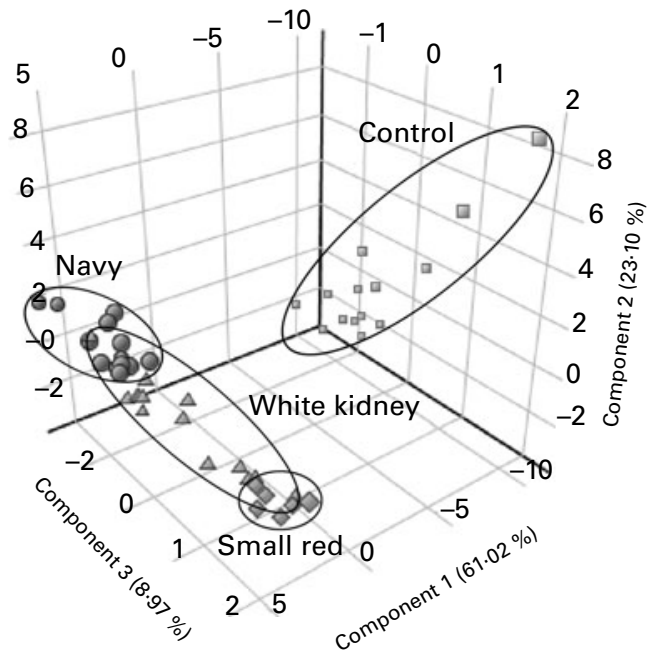

Fig. 5. Principal component analysis (PCA) of the effect of bean diet on tumour tissue. (A) Electrospray ionisation (ESI)-positive, (B) ESI-negative. PCA shows a separate clustering based on control $(\square)$, white kidney $(\diamond)$, small red $(0)$ and navy $(\Delta)$ bean diets.

\section{Discussion}

There is currently a resurgence of interest in encouraging increased consumption of pulses such as dry beans. However, newly released dietary guidelines from the US Department of Agriculture do not support this trend, in part because Americans have failed to achieve the previously recommended amounts $^{(25)}$. Rather, the new guidelines suggest that pulses be consumed in reduced amounts in comparison with previous guidelines $^{(26)}$. While this seems surprising at first glance, a more careful review of the evidence reveals a body of literature that is supportive of pulse consumption but that fails to make a compelling case for their inclusion in the diet, in part due to known barriers to eating beans and to a lack of solid mechanistic data that account for health benefits ${ }^{(9,16-18)}$. In the present

Table 2. Metabolites tentatively identified in mammary carcinomas*

\begin{tabular}{|c|c|c|c|c|}
\hline $\mathrm{RT}(\min )$ & Mass (Da) & Mode & Trend† & Metabolite typeł \\
\hline 0.91 & 85.0532 & $\mathrm{ESI}+$ & $\uparrow$ & Carboxylic acid \\
\hline 0.98 & $122 \cdot 0477$ & $\mathrm{ESI}+$ & $\uparrow$ & Carboxylic acid \\
\hline 1.70 & $205 \cdot 1314$ & $\mathrm{ESI}+$ & $\uparrow$ & Primary alcohol \\
\hline 1.79 & 99.0689 & $\mathrm{ESI}+$ & $\uparrow$ & Carboxylic acid \\
\hline $3 \cdot 10$ & $259 \cdot 1782$ & $\mathrm{ESI}+$ & $\uparrow$ & Fatty acyl carnitine \\
\hline 3.87 & $142 \cdot 0995$ & $\mathrm{ESI}+$ & $\uparrow$ & Unsaturated fatty acid \\
\hline 5.01 & $172 \cdot 1095$ & $\mathrm{ESI}+$ & $\uparrow$ & Oxo fatty acid (hydroxy fatty acid) \\
\hline $5 \cdot 12$ & $284 \cdot 1830$ & $\mathrm{ESI}+$ & $\downarrow$ & Secondary amine \\
\hline $5 \cdot 38$ & $116 \cdot 0835$ & $\mathrm{ESI}+$ & $\uparrow$ & Methyl branched fatty acid \\
\hline $5 \cdot 38$ & 335.0796 & $\mathrm{ESI}+$ & $\uparrow$ & Primary amine \\
\hline $6 \cdot 34$ & $246 \cdot 0857$ & $\mathrm{ESI}+$ & $\uparrow$ & Secondary alcohol \\
\hline $7 \cdot 63$ & $200 \cdot 1411$ & $\mathrm{ESI}+$ & $\uparrow$ & Oxo fatty acid (hydroxy fatty acid) \\
\hline 7.63 & $144 \cdot 1149$ & $\mathrm{ESI}+$ & $\uparrow$ & Methyl branched fatty acid \\
\hline $7 \cdot 63$ & $126 \cdot 1044$ & $\mathrm{ESI}+$ & $\uparrow$ & Fatty aldehyde \\
\hline 7.96 & $246 \cdot 1211$ & $\mathrm{ESI}+$ & $\uparrow$ & Primary amine \\
\hline $8 \cdot 26$ & $350 \cdot 2090$ & $\mathrm{ESI}+$ & $\uparrow$ & Prostaglandin E series \\
\hline $8 \cdot 66$ & $444 \cdot 2694$ & $\mathrm{ESI}+$ & $\uparrow$ & Prostaglandin F series \\
\hline 9.04 & $440 \cdot 1580$ & $\mathrm{ESI}+$ & $\uparrow$ & Primary amine \\
\hline $9 \cdot 30$ & $210 \cdot 1969$ & ESI - & $\downarrow$ & Fatty aldehyde \\
\hline 9.55 & $398 \cdot 2070$ & ESI - & $\downarrow$ & Bile acids, alcohols and derivatives \\
\hline $9 \cdot 64$ & $486 \cdot 2766$ & $\mathrm{ESI}+$ & $\uparrow$ & Dialkyl ether \\
\hline 11.45 & $242 \cdot 2240$ & $\mathrm{ESI}+$ & $\downarrow$ & Methyl branched fatty acid \\
\hline 11.45 & $210 \cdot 1971$ & $\mathrm{ESI}+$ & $\downarrow$ & Fatty aldehyde \\
\hline $12 \cdot 08$ & $256 \cdot 2412$ & $\mathrm{ESI}+$ & $\downarrow$ & Methyl branched fatty acid \\
\hline $12 \cdot 37$ & $442 \cdot 1991$ & $\mathrm{ESI}+$ & $\uparrow$ & Diacylglycerophosphoglycerol \\
\hline 12.58 & $284 \cdot 2723$ & $\mathrm{ESI}+$ & $\downarrow$ & Methyl branched fatty acid \\
\hline 12.58 & $266 \cdot 2608$ & $\mathrm{ESI}+$ & $\downarrow$ & Fatty aldehyde \\
\hline $12 \cdot 62$ & $530 \cdot 2501$ & $\mathrm{ESI}+$ & $\uparrow$ & Monoacylglycerophosphoinositol \\
\hline
\end{tabular}

$\mathrm{RT}$, retention time; ESI + , electrospray ionisation positive mode; ESI - , electrospray ionisation negative mode. * List based on MassTRIX query of Kyoto Encyclopedia of Genes and Genomes (KEGG).

$\dagger$ Indicates feature is increased in bean-fed animals $(\uparrow)$ or decreased in bean-fed animals $(\downarrow)$ relative to the control group.

$\ddagger$ Metabolite type assigned based on KEGG search using MassTRIX. Total number of masses searched $=350$, metabolite types were identified $=28 / 350$ or $8.0 \%$, non-identified metabolite types $=322 / 350$ or $92.0 \%$ (not shown). 


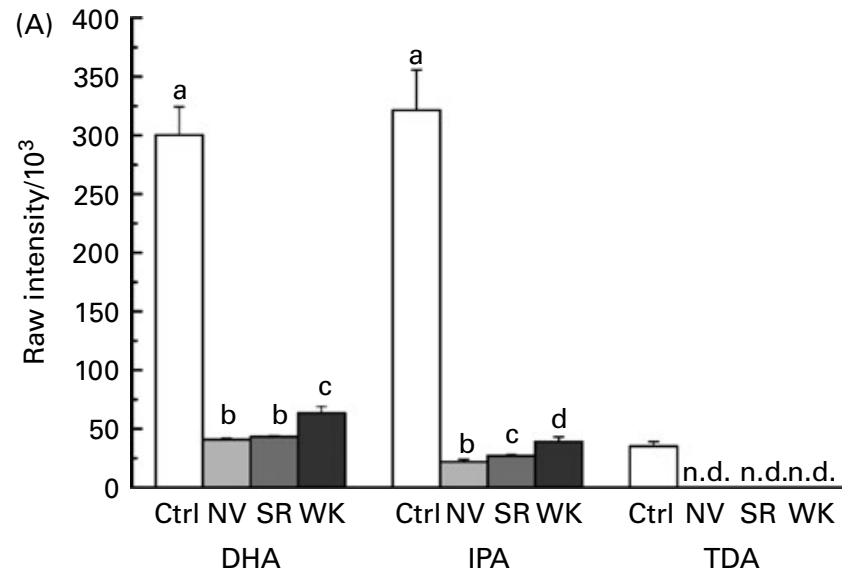

Methyl branched fatty acids

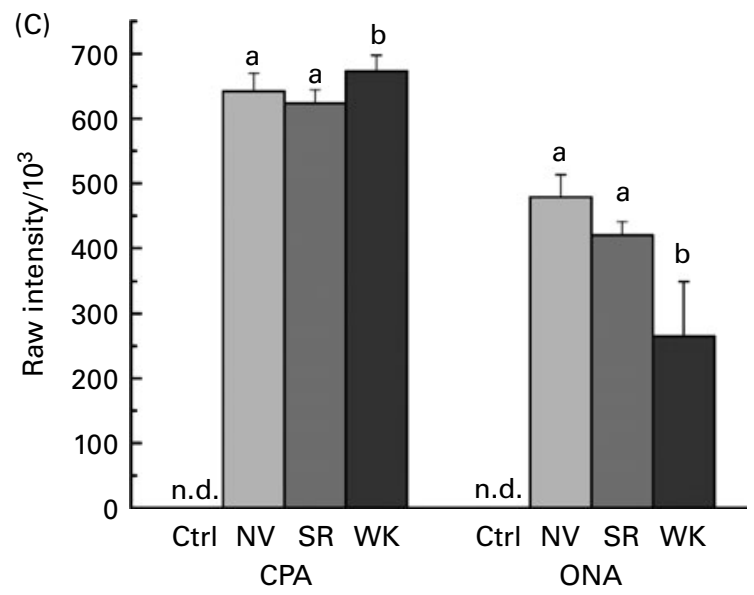

Oxo fatty acids

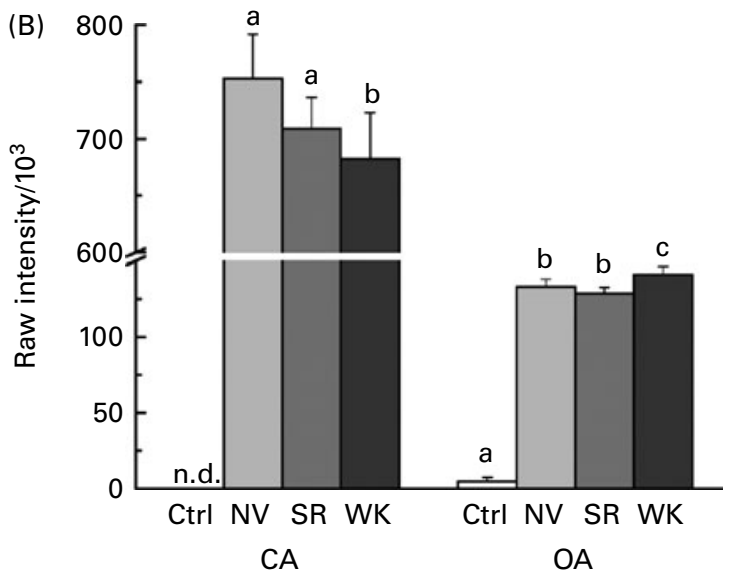

Straight chain fatty acids

(D)

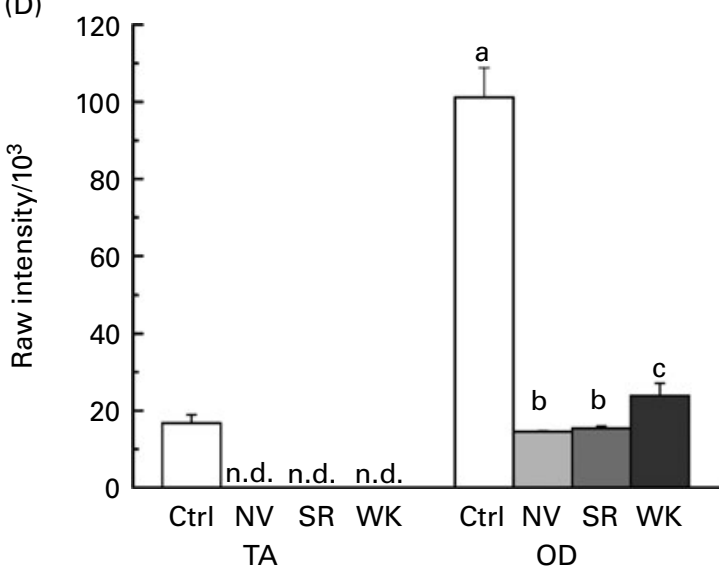

Fatty aldehydes

Fig. 6. Effect of bean diet on fatty acyl compounds in mammary carcinomas. (A) Methyl branched fatty acids, (B) straight-chain fatty acids, (C) oxo fatty acids, (D) fatty aldehydes. Data are shown as fold change relative to control. Values are means of at least nine animals per group $(n 9-10)$, with standard errors represented by vertical bars. Metabolite intensity (relative concentration) was measured using liquid chromatography-time-of-flight MS and analysed using KruskalWallis one-way ANOVA. ${ }^{\text {a,b,c,d }}$ Mean values with unlike letters were significantly different $(P<0.05)$. n.d., Not detected; Ctrl, control; NV, navy bean-fed; SR, small red bean-fed; WK, white kidney bean-fed; DHA, 2,14-dimethyl hexanoic acid; IPA, isopalmitic acid; TDA, 3,7,11-trimethyl docecanoic acid; CA, caproic acid; OA, octanoic acid; CPA, 3-capryl propionic acid; ONA, 8-oxo-nonanoic acid; TA, 2-tradecenal; OD, 9-octadecenal.

paper, we used metabolomic profiling as a novel approach to test the global hypothesis that dry bean consumption promotes health through the regulation of lipid metabolism ${ }^{(27)}$. While evidence for this hypothesis has emerged from several sources, to our knowledge this represents the first report of the modulation of lipid metabolism by dry beans using an 'omics' approach. As discussed in the following paragraphs, the data obtained clearly indicate that lipid metabolism is modified in both mammary gland and mammary carcinomas of bean-fed rats relative to rats fed a control (bean-free) diet, and that argue additional experiments are warranted to identify specific gene targets that account for the observed effects on lipid metabolism. The data reported also are consistent with variation among bean genotypes in the traits that alter lipid metabolism and thus indicate the potential for plant scientists to improve dry beans through classical approaches used in plant breeding and selection.
Metabolomics is a high-throughput screening tool that lends itself to hypothesis generation at the level of cells or tissues ${ }^{(28)}$. While the metabolomics field is still limited in its application to the identification of natural products and bioactive food components in plant materials because of the libraries that are currently available for ion identification, the compound libraries for mammalian metabolites are sufficiently developed to permit tentative identification and association with the metabolic pathways $^{(29)}$. Accordingly, Tables 1 and 2 contain lists of ions to which identities were assigned; these identities are tentative because of the need to analyse validated standards, assess percentage recovery, and perform standard curve experiments for quantitative assessments using the conditions of chromatography and mass analysis defined in the Methods section. Validation of compound identification is considered a next step in this programme of research. Nonetheless, the qualitative data shown in Tables 1 and 2 clearly indicate that dry bean 
consumption has effects on many lipid metabolites and therefore the cellular pathways responsible for their synthesis, interconversion and/or degradation.

Many approaches can be taken to analyse and interpret metabolomics data and although there are clearly on-going efforts to create a uniform framework for analysis ${ }^{(30,31)}$, a standardised method does not currently exist. Our protocol was: (1) to filter data according to criteria that permitted generation of a 'high-quality' ion database consisting of ion masses and retention times; (2) to subject that list to statistical analysis to generate a sublist of ions that differed significantly between treatment groups; (3) to submit the ion list from point 2 to unsupervised PCA, to ask whether treatment with dietary beans affected the metabolome of the mammary gland (Fig. 1) or the mammary carcinomas (Fig. 5) that occurred in control $v$. bean-fed rats; and (4) to submit the list of masses used in PCA to MassTRIX for tentative ion identification and association with metabolic pathways (Tables 1 and 2). As noted in the Results section, separation between control and bean-fed rats was observed in both mammary gland and mammary carcinomas. This finding represents a critical proof-of-concept that eating a dry beancontaining diet exerts effects on mammalian cellular metabolism in two peripheral tissues with markedly different cellular composition. Mammary gland is a tissue that is primarily comprised of adipocytes; however, mammary carcinomas are primarily comprised of epithelial cells. Both tissues types have a heterogeneous mixture of cell types, but mammary gland is physiologically adapted for milk production; however, mammary carcinomas are pathologies, the cells of which display altered metabolic characteristics as outlined in Wood et al. ${ }^{(32)}$.

With the proof-in-principle evidence in hand, the same mass lists used in the PCA analyses were submitted to MassTRIX ${ }^{(33)}$. This analysis tool links ion masses to a database of candidate compound identities also linked to the KEGG metabolic pathways. MassTRIX analysis was first completed for mammary gland and the resulting list of hits for the masses entered is provided in Table 1. Most noteworthy was the prominent identification of lipid metabolites as being up- or down-regulated in the bean-fed diet groups relative to the control diet-fed group (Figs. 2-4). KEGG links were identified to lipid biosynthesis, steroid biosynthesis and intermediary metabolism of lipids. This finding suggests that bioactive components of beans are likely to be affecting key regulatory elements in lipid metabolism and supports evaluating the effects of dry bean consumption on enzyme loci such as acetyl CoA carboxylase, fatty acid synthase, and 3-hydroxy-3-methyl-glutaryl (HMG)-CoA reductase, key regulators of lipid metabolism. It should be noted that other lipid-metabolising enzymes are also likely to be involved.

With evidence that mammary gland lipid metabolism was affected by bean consumption, the metabolomics database for mammary carcinomas was then submitted to MassTRIX despite the fact that a carcinoma in the bean-treated group reflected the failure of beans to inhibit carcinoma development. The analysis of carcinomas in this model system has been useful in previous experiments in garnering clues about mechanisms of effect. The masses for which tentative compound identification were provided by MassTRIX are shown in Table 2. As with normal mammary gland, lipid metabolism was highly enriched in the pathways identified. However, there were differences in the nature of the pathways implicated. For example, diacylglycerol was lower in the normal mammary glands of bean-fed rats; alterations in prostaglandin metabolism were implied in the mammary carcinomas. This is likely to be informative since differential regulation of eicosanoid synthesis and degradation is known to be involved in the genesis and progression of cancer $^{(34)}$

One goal of the present study was to develop hypotheses about mechanisms that could account for the inhibition of mammary carcinogenesis that we have previously reported ${ }^{(14,15)}$ The results of the analyses of normal mammary gland and mammary carcinomas provided the rationale for two hypotheses The mammary gland data are consistent with the hypothesis that bean feeding down-regulates formation of diacylglycerols and related intracellular messengers which affect signalling by either protein kinase $\mathrm{C}$ or the nutrient-sensing master control protein, mammalian target of rapamycin (mTOR) ${ }^{(35,36)}$ Negative regulation of the pathways of these proteins and components has been reported to be a mechanism of cancer prevention, whereas misregulation of mTOR has been implicated in the development of cancer. Our second hypothesis is based on the data from the mammary carcinomas that we have reported to be markedly smaller in bean-fed relative to control-fed rats. We hypothesise that bean consumption modulates eicosanoid metabolism in mammary carcinomas and that this results in lower tumour mass by changing the ratios of eicosanoids within the tumour. From the viewpoint of metabolic regulation, this implicates involvement of cyclo-oxygenase-2, the rate-limiting step in eicosanoid degradation which is catalysed by prostaglandin dehydrogenase, a catabolic enzyme that serves a potent tumour suppressor function ${ }^{(37-39)}$ In addition to these ideas, it is important to consider that rapidly dividing tumour cells require robust de novo synthesis of lipids as part of the metabolic reprogramming that is essential for the development and progression of cancer ${ }^{(40,41)}$. Given that bean consumption appears to target lipid metabolism, investigation of the effects on the reprogramming of metabolism that occurs in tumours is warranted since bean consumption could serve to enhance the efficacy of cancer therapy as well as play a role in cancer prevention.

Each type of bean that was studied is representative of a distinct dry bean market class. Bean market classes share comparable seed size, shape, colour and texture but are actually collections of similar genotypes within specific gene pools ${ }^{(42)}$ Market classes arose during domestication as a result of human selection for plant morphology and seed characteristics leading to modern-day differences among seed types. Bean market classes are further organised by race and most broadly by centre of domestication (COD $)^{(43,44)}$. The COD of an agricultural crop denotes the location where domestication took place. The SR bean is a market class in the Middle American COD, race - Durango, the NV bean is also a market class in the Middle American COD, but race - Mesoamerican, and the WK market class is from the Andean COD, race - Nueva Granada. Beans from these races account for more than $80 \%$ of worldwide common bean production ${ }^{(44)}$. Knowing that these 
bean types represent distinct genetic heritage, an additional question addressed was whether these genetic distinctions translated into differential effects on tissue metabolomes. The PCA analyses shown in Figs. 1 and 4 provide evidence that these genotypically distinct dry bean market classes caused distinguishable differences in the mammary gland and the mammary carcinoma metabolomes, specifically in lipid metabolites (Tables 1-2). While this finding has many implications, a practical value is that these differences will serve to provide wellmatched positive and negative controls for detailed analyses to determine how dry bean components regulate genes that control lipid biosynthesis and catabolism.

In summary, mounting evidence indicates that the decline in dry bean consumption that has occurred during global industrialisation and economic development may have associations with the rise in chronic disease rates that have accompanied the pandemic of obesity. The effects that dry beans may exert on chronic disease mechanisms are diverse and have not yet been extensively investigated. The evidence presented here identifies lipid metabolism as a critical target of bean bioactive food components for future studies with a focus on determining how dry bean consumption affects key regulators of lipid biosynthesis, interconversion and degradation.

\section{Acknowledgements}

The present study was supported in part by USAID grant no. REE-A-00-03-00094-00, the Bean Health Alliance, the American Institute for Cancer Research grant no. 08A032, and the US Department of Agriculture National Institute of Food and Agricultural Agriculture and Food Research Initiative Project no. 2009-01929. The authors thank Steven Fischer, Agilent Technologies for his assistance with the analytical work, Mark Brick for editorial assistance, and Erica Danielle, Vanessa Fitzgerald, Elizabeth Neil, and Jennifer Sells for their excellent technical assistance. M. M. M. conducted the metabolomics analyses including data evaluation. J. N. M. participated in various aspects of study implementation and data evaluation and interpretation. H. J. T. designed the carcinogenesis study and provided oversight of all aspects of the experiment. All authors participated in writing the manuscript. There are no conflicts of interest.

\section{References}

1. FAOSTAT \& Food and Agriculture Organization (2009) FAOSTAT http://faostat.fao.org

2. Geil PB \& Anderson JW (1994) Nutrition and health implications of dry beans: a review. J Am Coll Nutr 13, 549-558.

3. Gepts P (2004) Crop domestication as a long-term selection experiment. Plant Breeding Rev 24, 1-44.

4. Leterme P (2002) Recommendations by health organizations for pulse consumption. Br J Nutr 88, Suppl. 3, S239-S242.

5. Mitchell DC, Lawrence FR, Hartman TJ, et al. (2009) Consumption of dry beans, peas, and lentils could improve diet quality in the US population. J Am Diet Assoc 109, 909-913.

6. World Health Organization (2008) Chronic disease http:// www.who.int/topics/chronic_diseases/en/
7. World Health Organization (2008) Preventing chronic diseases: a vital investment http://www.who.int/chp/chronic_ disease_report/en/

8. Thompson MD \& Thompson HJ (2009) Biomedical agriculture: a systematic approach to food crop improvement for chronic disease prevention. In Advances in Agronomy, pp. 1-54 [D Sparks, editor]. San Diego, CA: Academic Press.

9. Bennink MR \& Rondini E (2008) Beans and Health: A Comprehensive Review. Frazee, MN: The Bean Institute.

10. Eyre H, Kahn R \& Robertson RM (2004) Preventing cancer, cardiovascular disease, and diabetes: a common agenda for the American Cancer Society, the American Diabetes Association, and the American Heart Association. CA Cancer J Clin 54, 190-207.

11. Frasca F, Pandini G, Sciacca L, et al. (2008) The role of insulin receptors and IGF-I receptors in cancer and other diseases. Arch Physiol Biochem 114, 23-37.

12. Hartman TJ, Albert PS, Zhang ZY, et al. (2010) Consumption of a legume-enriched, low-glycemic index diet is associated with biomarkers of insulin resistance and inflammation among men at risk for colorectal cancer. J Nutr 140, 60-67.

13. Zhang ZY, Lanza E, Kris-Etherton PM, et al. (2010) A high legume low glycemic index diet improves serum lipid profiles in men. Lipids 45, 765-775.

14. Thompson MD, Brick MA, McGinley JN, et al. (2009) Chemical composition and mammary cancer inhibitory activity of dry bean. Crop Sci 49, 179-186.

15. Thompson MD, Thompson HJ, Brick MA, et al. (2008) Mechanisms associated with dose-dependent inhibition of rat mammary carcinogenesis by dry bean (Phaseolus vulgaris, L.). J Nutr 138, 2091-2097.

16. Duane WC (1997) Effects of legume consumption on serum cholesterol, biliary lipids, and sterol metabolism in humans. J Lipid Res 38, 1120-1128.

17. Farrell DJ, Girle L \& Arthur J (1978) Effects of dietary fibre on the apparent digestibility of major food components and on blood lipids in men. Aust J Exp Biol Med Sci 56, 469-479.

18. Guillon F \& Champ MM (2002) Carbohydrate fractions of legumes: uses in human nutrition and potential for health. Br J Nutr 88, Suppl. 3, S293-S306.

19. Thompson HJ, Singh M \& McGinley J (2000) Classification of premalignant and malignant lesions developing in the rat mammary gland after injection of sexually immature rats with 1-methyl-1-nitrosourea. J Mammary Gland Biol Neoplasia 5, 201-210.

20. Sana TR, Waddell K \& Fischer SM (2008) A sample extraction and chromatographic strategy for increasing LC/MS detection coverage of the erythrocyte metabolome. J Chromatogr B Anal Technol Biomed Life Sci 871, 314-321.

21. Hendriks G, Uges DRA \& Franke JP (2007) Reconsideration of sample $\mathrm{pH}$ adjustment in bioanalytical liquid-liquid extraction of ionisable compounds. J Chromatogr B Anal Technol Biomed Life Sci 853, 234-241.

22. Benjamini Y \& Hochberg Y (1995) Controlling the false discovery rate: a practical and powerful approach to multiple testing. J R Statist Soc B 57, 289-300.

23. Sokal RR \& Rohlf FJ (1995) Biometry: The Principles and Practice of Statistics in Biological Research. New York: W.H. Freeman.

24. Hochberg Y \& Tamhane AC (1987) Multiple Comparision Procedures. New York: John Wiley \& Sons, Inc.

25. US Department of Agriculture (2010) Dietary Guidelines http://www.cnpp.usda.gov/DietaryGuidelines.htm

26. US Department of Health and Human Services (2005) Dietary Guidelines for Americans www.healthierus.gov/dietaryguidelines 
27. Gibney MJ, Walsh M, Brennan L, et al. (2005) Metabolomics in human nutrition: opportunities and challenges. Am J Clin Nutr 82, 497-503.

28. Weckwerth W (2010) Metabolomics: an integral technique in systems biology. Bioanalysis 2, 829-836.

29. Tohge T \& Fernie AR (2009) Web-based resources for massspectrometry-based metabolomics: a user's guide. Phytochemistry 70, 450-456.

30. Kind T \& Fiehn O (2007) Seven golden rules for heuristic filtering of molecular formulas obtained by accurate mass spectrometry. BMC Bioinformatics $\mathbf{8}, 105$.

31. Scalbert A, Brennan L, Fiehn O, et al. (2009) Mass-spectrometrybased metabolomics: limitations and recommendations for future progress with particular focus on nutrition research. Metabolomics 5, 435-458.

32. Wood LD, Parsons DW, Jones S, et al. (2007) The genomic landscapes of human breast and colorectal cancers. Science 318, 1108-1113.

33. Suhre K \& Schmitt-Kopplin P (2008) MassTRIX: mass translator into pathways. Nucleic Acids Res 36, W481-W484.

34. Wang D \& Dubois RN (2010) Eicosanoids and cancer. Nat Rev Cancer 10, 181-193.

35. Laplante M \& Sabatini DM (2009) An emerging role of mTOR in lipid biosynthesis. Curr Biol 19, R1046-R1052.
36. Sakane F, Imai S, Kai M, et al. (2008) Diacylglycerol kinases as emerging potential drug targets for a variety of diseases. Curr Drug Targets 9, 626-640.

37. Muller-Decker K \& Furstenberger G (2007) The cyclooxygenase-2-mediated rostaglandin signaling is causally related to epithelial carcinogenesis. Mol Carcinog 46, 705-710.

38. Wang D \& Dubois RN (2004) Cyclooxygenase-2: a potential target in breast cancer. Semin Oncol 31, 64-73.

39. Wolf I, O'Kelly J, Rubinek T, et al. (2006) 15-Hydroxyprostaglandin dehydrogenase is a tumor suppressor of human breast cancer. Cancer Res 66, 7818-7823.

40. Jones RG \& Thompson CB (2009) Tumor suppressors and cell metabolism: a recipe for cancer growth. Genes Dev $\mathbf{2 3}$, 537-548

41. Kaelin WG \& Thompson CB (2010) Q\&A: cancer: clues from cell metabolism. Nature 465, 562-564.

42. Kami J, Velasquez VB, Debouck DG, et al. (1995) Identification of presumed ancestral DNA-sequences of phaseolin in Phaseolus vulgaris. Proc Natl Acad Sci US A 92, 1101-1104.

43. Singh SP (2001) Broadening the genetic base of common bean cultivars: a review. Crop Sci 41, 1659-1675.

44. Singh SP, Gepts P \& Debouck DG (1991) Races of common bean (Phaseolus vugaris L.). Econ Bot 45, 379-396. 